MIGRACIONES INTERNACIONALES, VOL. 11, ART. 11, 2020 e-ISSN 2594-0279 http://dx.doi.org/10.33679/rmi.v1i1.1731

\title{
Back on your own: migración de retorno y la respuesta del gobierno federal en México
}

\section{Back on your Own: Return Migration and the Federal Government Response in Mexico}

\author{
Mónica Liliana Jacobo Suárez y Nuty Cárdenas Alaminos ${ }^{2}$
}

\section{RESUMEN}

La situación migratoria México-Estados Unidos se ha transformado por completo. Más mexicanos retornan de Estados Unidos a México que aquellos que migran a ese país. Millones de mexicanos han salido de Estados Unidos debido a la recesión económica, el endurecimiento de la política migratoria y el fortalecimiento del sistema de deportaciones estadounidense. Los retornados, voluntarios e involuntarios, presentan un perfil diverso y necesidades de reintegración variadas, lo que constituye un desafío para México. Aquí analizamos las iniciativas y programas creados por el gobierno federal mexicano para atender a esta población. Identificamos varios desafíos y áreas de oportunidad y concluimos con recomendaciones para mejorar el proceso de reintegración.

Palabras clave: 1. migración de retorno, 2. reintegración, 3. política migratoria, 4. México, 5. Estados Unidos.

\section{ABSTRACT}

U.S.-Mexico migration has been completely transformed. Currently, more Mexicans return from the United States to Mexico than those who migrate to that country. Millions of Mexican migrants have left the United States due to the economic recession, a harsher immigration policy, and a stronger deportation system. Mexican returnees, voluntary and involuntary, present a diverse profile and wide-ranging reintegration needs, which constitutes a challenge for the government in Mexico. Here we analyze specific initiatives and programs created by the Mexican federal government to serve returnees, also we identify various challenges and areas of improvement. Finally, we offer recommendations for a better reintegration of the returnee population.

Keywords: 1. return migration, 2. reintegration, 3. migration policy, 4. Mexico, 5. United States.

Fecha de recepción: 30 de agosto de 2017

Fecha de aceptación: 10 de abril de 2019

Publicación en web: 15 de julio de 2020

\footnotetext{
${ }^{1}$ Centro de Investigación y Docencia Económicas, México, monica.jacobosuarez@gmail.com, https://orcid.org/0000-0001-8487-5266

${ }^{2}$ Centro de Investigación y Docencia Económicas, México, nuty.cardenas@cide.edu, https://orcid.org/0000-0002-4034-8111
} 


\section{INTRODUCCIÓN}

Los patrones de migración a nivel mundial han sufrido grandes transformaciones durante la última década. Dada la crisis financiera de 2008 y las políticas restrictivas de inmigración en los estados receptores, el retorno forzado e involuntario se ha convertido ahora en una característica destacada de los flujos migratorios internacionales. Ese es el caso del que solía ser el principal corredor migratorio del mundo, la frontera México-Estados Unidos (Durand y Arias, 2014), que actualmente está caracterizado por un número significativo de mexicanos que regresan al país de origen. Aunque este no es un fenómeno nuevo en México, pues un número considerable de mexicanos fueron deportados de los Estados Unidos en los años 30 (Alanís, 2015), la migración de retorno ha crecido en importancia debido a su volumen, la variedad de experiencias y la urgencia de tener programas para reintegrar a esta población.

Si bien ha habido un avance importante en la caracterización de la población retornada (Fundación Bancomer BBVA, 2016; Gandini, Lozano y Gaspar, 2015; Masferrer y Roberts, 2016), la investigación sobre las necesidades de reintegración de los retornados es escasa (UNICEF, 2015; Guzmán, 2014) y los análisis sobre los programas gubernamentales para atenderlas son casi nulos (García y Gaspar, 2017) a pesar de los 2.8 millones de remociones a México realizadas bajo la administración de Obama.

Actualmente, diseñar programas de atención a la población de retorno es complejo, pues a diferencia de flujos de retorno previo, el retorno en las últimas dos décadas incluye una diversidad de perfiles migratorios. Una primera categoría, el retorno forzado, la conforman aquellos connacionales que salen de Estados Unidos a través de procesos de remoción (removal), o de una orden de retorno voluntario (return). En general, a su entrada a México estos migrantes se apegan a procesos de repatriación, lo que permite contabilizarlos. Fuera del flujo de retorno forzado, aunque vinculado con este, se encuentran los niños y los cónyuges que siguen a un familiar deportado. Estas personas generalmente no se incluyen en las estadísticas de repatriación y remoción, ya que su regreso se considera "voluntario".

Dentro del retorno voluntario se incluyen también aproximadamente 500000 menores nacidos en Estados Unidos e hijos de migrantes mexicanos, para los cuales comúnmente el mudarse a México no representa un retorno, sino un primer movimiento migratorio. Por tanto, las necesidades de la población retornada son múltiples y diversas, en particular entre aquellos que regresan de manera forzada y por lo tanto, no pueden planificar su regreso. A menudo, la población deportada carece de documentos de identidad vigentes o válidos, como por ejemplo certificados de nacimiento o la credencial para votar, esta última suele ser considerada como el documento de identidad oficial requerido para acceder a casi todos los servicios en México (Mexicans and Americans Thinking Together, 2013; Medina y Menjívar, 2015).

Este artículo busca ampliar el conocimiento sobre el fenómeno de la migración de retorno en México, analizando las respuestas que el gobierno federal ha tenido para atender 
las necesidades de sus ciudadanos que regresan de Estados Unidos. La discusión está organizada alrededor de tres preguntas: ¿En qué consisten las principales iniciativas federales en materia de migración de retorno? ¿Cuál ha sido el alcance y las limitaciones de dichas iniciativas? ¿Qué acciones podrían contribuir a una mejor reintegración de la población de retorno a partir de las iniciativas gubernamentales existentes? En específico, discutimos los programas y regulaciones en materia migratoria y de retorno creados en la última década por el gobierno federal. Nuestro objetivo al analizar el funcionamiento y el alcance de dichas iniciativas es detectar los desafíos y las áreas de oportunidad.

Como caso de estudio, México tiene gran relevancia para el continente americano pues constituye el país con el mayor número absoluto de migrantes deportados desde Estados Unidos, aún el principal destino migratorio en el mundo (World Economic Forum, 2019). El discurso hostil hacia los migrantes, en particular hacia aquellos de origen mexicano por parte de Donald Trump, actual presidente de Estados Unidos, pone presión adicional para que el gobierno mexicano analice su respuesta hacia la población en retorno.

En la siguiente sección discutimos la literatura existente en torno al perfil de los migrantes de retorno, voluntarios e involuntarios, en las últimas dos décadas con el fin de identificar sus principales necesidades de reintegración. Posteriormente, examinamos las iniciativas gubernamentales encaminadas a atender a los retornados durante las administraciones de los presidentes Felipe Calderón Hinojosa (2006-2012) y Enrique Peña Nieto (2012-2018). Finalmente, concluimos con el análisis de estas respuestas gubernamentales e identificamos las principales deficiencias y ventanas de oportunidad en el actual contexto binacional México-Estados Unidos.

\section{EL RETORNO DE LOS MIGRANTES MEXICANOS EN EL SIGLO XXI}

La migración mexicana hacia Estados Unidos constituye un caso único en la historia de la migración internacional debido a que la vecindad e historia compartida entre estos dos países han desencadenado flujos de inmigrantes mexicanos que se dirigen al norte (Durand, 2000). De hecho, hasta 2014 (Hazán, 2014) México fue el mayor proveedor de inmigrantes a Estados Unidos. No obstante, el flujo de mexicanos que migra a Estados Unidos ha variado en su magnitud durante los siglos XX y XXI. A cada flujo migratorio hacia los Estados Unidos le corresponde un flujo de mexicanos que regresan al país de origen.

El primer flujo masivo de mexicanos que regresaron a su país desde Estados Unidos ocurrió durante la Gran Depresión; cientos de miles de inmigrantes de origen mexicano fueron expulsados entre 1930 y 1933. El gobierno mexicano reaccionó con críticas a las deportaciones y anunció una serie de programas dirigidos a la reintegración de los connacionales, aunque la mayoría de estas iniciativas fracasaron debido a la falta de financiamiento y una implementación deficiente (Alanís, 2015). Posteriormente, México recibió a un número importante de connacionalesdurante la Operación Espalda Mojada 
(1954) mediante la cual se aprehendieron y deportaron a más de un millón de mexicanos indocumentados (Durand, 2016).

En el siglo XXI nos encontramos en una etapa de la migración México-Estados Unidos caracterizada por un mayor número de mexicanos que regresan de Estados Unidos a México, que aquellos que migran a ese país. Entre los cientos de miles que han regresado se observa el retorno voluntario y el regreso forzado. Este último retorno lo conforman aquellos connacionales que salen de Estados Unidos a través de procesos de remoción (removal) o una orden de retorno voluntario (return). Un migrante removido es una persona extranjera que es inadmisible o es deportada a México desde Estados Unidos. Su salida es obligatoria y confirmada mediante una orden de retiro bajo la modalidad de "criminal" o "no criminal". En Estados Unidos, cualquier inmigrante que es removido -deportado- tiene consecuencias administrativas o criminales si reingresa al territorio posteriormente.

El gobierno estadounidense define al retorno como el movimiento confirmado de un extranjero inadmisible o deportable fuera de Estados Unidos, no basado en una orden de retiro (no forzoso). En general, estos migrantes se apegan a procesos de repatriación a su entrada a México, lo que permite contabilizarlos en las estadísticas que genera el gobierno mexicano. Fuera del flujo de retorno forzado, aunque vinculado con este, se encuentran los niños y los cónyuges que siguen a un familiar deportado. Generalmente no se incluye a estos mexicanos en las estadísticas de repatriación y remoción, ya que su regreso se considera "voluntario".

Por lo menos dos factores parecen explicar el regreso de aproximadamente 2.8 millones de connacionales a territorio mexicano. Por un lado, la crisis financiera y económica en Estados Unidos restringió las oportunidades laborales para los migrantes, sobre todo para los indocumentados. Por el otro, la aplicación de políticas migratorias restrictivas -tanto en el ámbito federal, como en el local- amplió drásticamente la base de la población que podía ser deportada y fortaleció la ejecución del sistema de deportación.

La aplicación de las leyes aprobadas en la década de los años noventa-Ley de Reforma de la Inmigración Ilegal y de Responsabilidad del Inmigrante (IIRIRA por sus siglas en inglés) y la Ley Antiterrorista y de Pena de Muerte Efectiva (AEDPA por sus siglas en inglés)- así como los cambios en la legislación y el control administrativo de la migración - con la creación del Departamento de Seguridad Nacional- a partir de los atentados de septiembre de 2001, fortalecieron el sistema de deportación a nivel federal estadounidense. Por ejemplo, se amplió la lista de delitos que conducen a la deportación. Las leyes también limitaron los motivos por los cuales una persona puede apelar un proceso de deportación y asignaron más fondos a la aplicación de la Ley de Inmigración (Hagan, Eschbach y Rodríguez, 2008).

Además, las alianzas entre las agencias de derecho federales, estatales y locales dieron prioridad al cumplimiento interno a través de los acuerdos 287 (g) y el programa 
Comunidades Seguras. La aplicación de la ley al interior del país intentó orillar a los inmigrantes indocumentados a las sombras haciendo que la vida cotidiana fuera muy difícil, para que optaran por la autodeportación (Hazán, 2014). De hecho, Comunidades Seguras, implementada entre 2008 y 2014 con el objetivo de priorizar la remoción de extranjeros criminales, fue bastante efectiva en la deportación de millones de hombres latinoamericanos, en su mayoría mexicanos.

Así, la implementación de la política migratoria en los Estados Unidos fue un importante impulsor del flujo de retorno a México. No sólo obligó a muchas personas a dejar el país a través de los procesos de deportación, sino que también presionó a los miembros de la familia (cónyuges e hijos en su mayoría) para que se reunieran con los repatriados en México (Hazán, 2014). Como consecuencia, los patrones de migración entre Estados Unidos y México se han transformado dramáticamente. En primer lugar, los Estados Unidos experimentaron una migración desde México neta "cero" de 2005 a 2010, un fenómeno que no se ha observado desde 1930 (Passel, D'Vera y González-Barrera, 2012). Posteriormente, la tendencia migratoria histórica se invirtió y desde 2015 más mexicanos regresan voluntaria e involuntariamente de los Estados Unidos, que los que emigran a ese país (GonzálezBarrera, 2015). Entre 2005 y 2010, 985000 mexicanos regresaron de Estados Unidos a México, cuatro veces el número registrado en 2000 (Escobar, Lowell y Martin, 2013).

Para medir el número de personas retornadas existen diversas fuentes (Gandini, et al 2015). En este caso mostramos los registros del gobierno de Estados Unidos y de México, mismos que sólo dan cuenta del retorno de las personas de manera forzada.

Figura 1. Mexicanos retornados y removidos de los Estados Unidos (2002-2016)

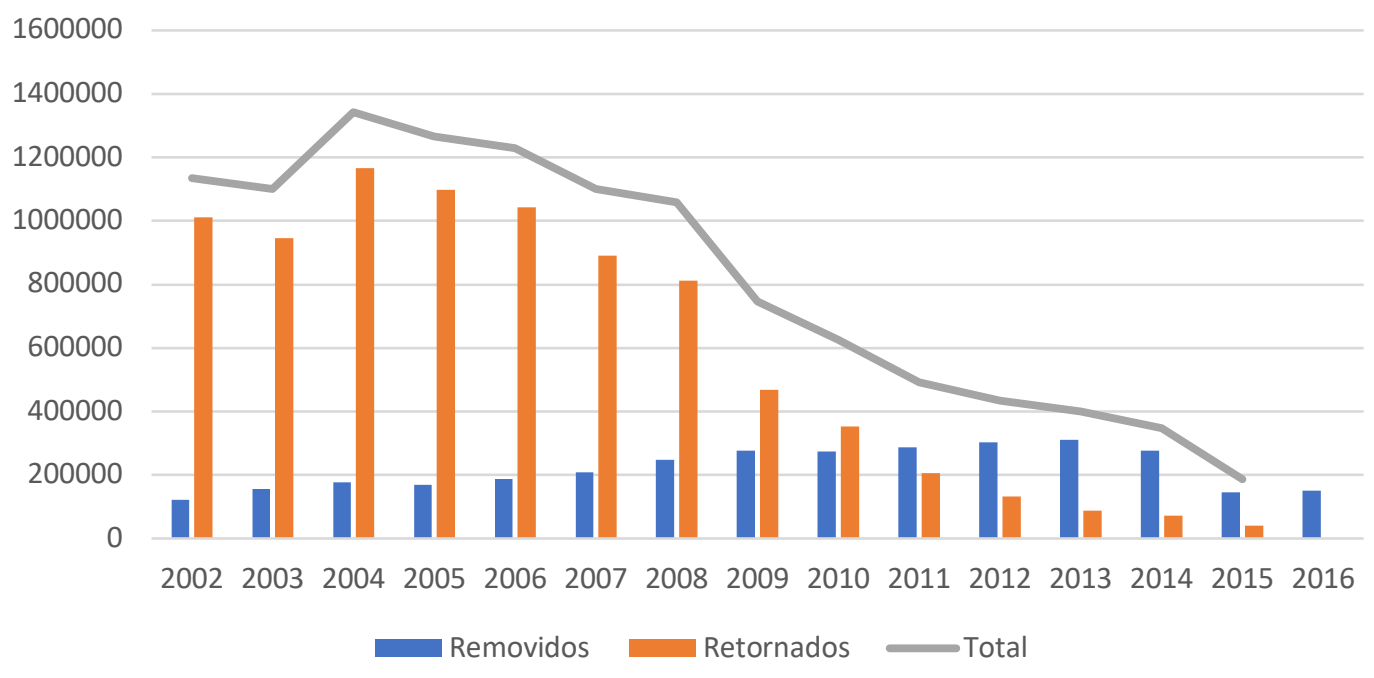

* No hay datos desagregados para los mexicanos retornados en 2016.

Fuente: Elaboración propia con base en datos del Department of Homeland Security (2015). 
Hay importantes tendencias a discutir. En primer lugar, el flujo general de retorno fue mayor durante la administración George W. Bush (2001-2009) que durante la del presidente Barak Obama (2009-2017). Aunque debemos tomar precaución, puesto que los retornos, la categoría que conforma la mayor parte del flujo en estos años, incluyen una minoría de nacionales de países distintos de México, pues así se reportan los datos para esos años. Sin embargo, según el Departamento de Seguridad Nacional de Estados Unidos, la mayoría de los retornos realizados entre 2002 y 2008 fueron ciudadanos mexicanos que habían sido aprehendidos por la Patrulla Fronteriza de los Estados Unidos.

En segundo lugar, cuando consideramos el retorno por tipo de proceso administrativo, existe una creciente importancia de las remociones o deportaciones a partir de 2008 y hasta 2014, cuando esta categoría comenzó a disminuir. Los años mencionados coinciden con la creación de Comunidades Seguras (2008) y su expansión a todo Estados Unidos en 2013, bajo el segundo periodo de la administración de Obama. Como un programa de deportación, Comunidades Seguras tenía como objetivo identificar y eliminar (desde el interior) delincuentes graves dentro de la población no autorizada.

Sin embargo, varios informes han criticado el funcionamiento del mencionado programa, ya que sólo una minoría de inmigrantes detenidos y deportados bajo Comunidades Seguras había cometido delitos graves. De hecho, la mayoría de los migrantes sujetos a procesos de deportación o remoción entre 2008 y 2013 no tienen antecedentes penales en absoluto o sólo han cometido delitos menores (Transactional Records Access Clearinghouse, 2013; The New York Times, 2014; Jacobo Suárez, 2014). En tercer lugar, las proporciones más grandes de mexicanos removidos desde interior se tradujeron en muchos jefes de familia que no tuvieron la oportunidad de planear su regreso y que, por consiguiente, fueron arrancados de su vida cotidiana. La alta probabilidad de que los migrantes que dejaron atrás una vida en los Estados Unidos sean seguidos por su familia (cónyuges e hijos) a México, implica más y mayores necesidades de reintegración.

Figura 2. Migrantes mexicanos repatriados según registros administrativos (2004-2016)

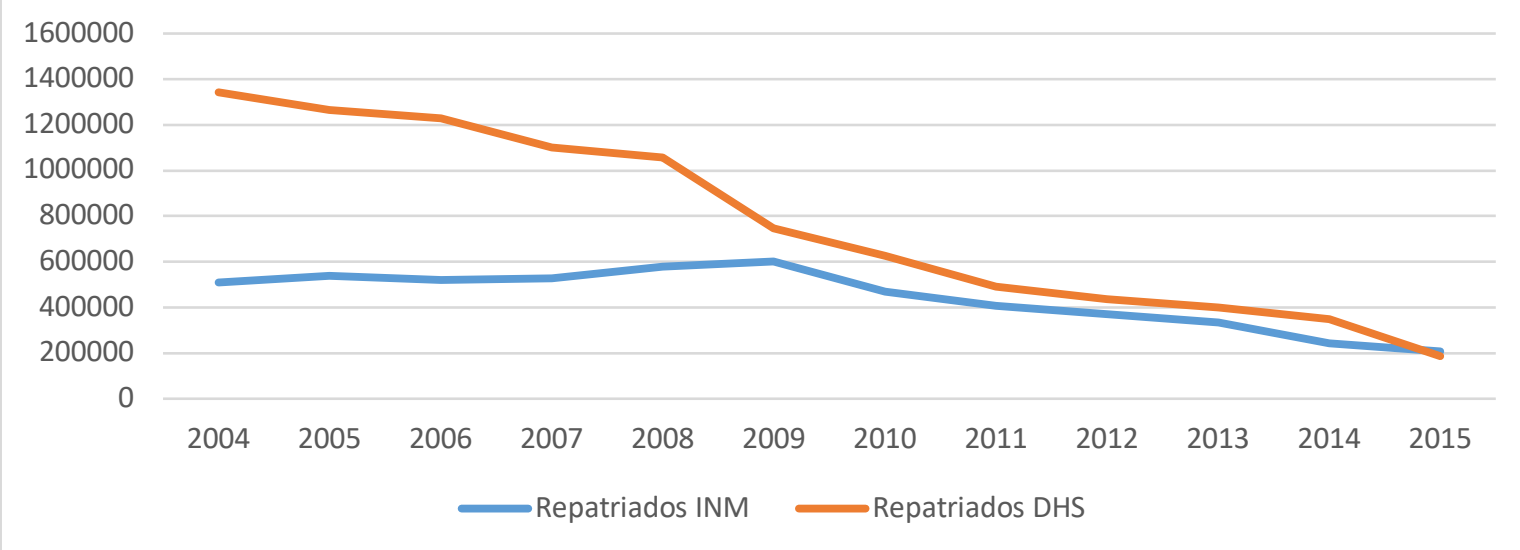

Fuente: Elaboración propia con datos de la Secretaría de Gobernación/Unidad de Política Migratoria (2004-2016) y del Department of Homeland Security (2015). 
Por último, es importante destacar que existe una diferencia entre los registros administrativos de migrantes de retorno que reporta el gobierno de Estados Unidos y los que contabiliza el gobierno de México (figura 2). De hecho, este último cuenta con un subregistro de la población retornada, pues sólo contabiliza a las personas repatriadas, es decir, aquellas que ingresaron por los puntos de entrada controlados por el Instituto Nacional de Migración.

Como explicaremos más adelante, lo anterior es en sí una limitante tanto para el entendimiento más completo del propio fenómeno del retorno, como para el diseño de políticas para la población retornada, pues sólo pueden ser beneficiarias de los programas ofrecidos por el gobierno federal mexicano aquellos que ingresaron por los puntos de repatriación y obtuvieron la constancia de repatriación. Los hijos y cónyugues que vienen siguiendo a sus familiares deportados y que entran de manera voluntaria a México no obtienen dicha constancia de repatriación y por tanto no son elegibles para los apoyos que ofrece el gobierno federal mexicano.

\section{CARACTERISTICAS DE LA POBLACIÓN DE RETORNO: ESTUDIOS RECIENTES}

¿Pero quiénes son los mexicanos que regresan a su país de origen? La diáspora de retorno está conformada por una diversidad de perfiles sociodemográficos por lo que las necesidades de integración pueden ser múltiples. Sin embargo, es posible identificar características generales entre la población retornada. En primer lugar, existe un importante grupo de personas en edad productiva y escolar dentro de la población de retorno, lo que resalta la importancia de crear mecanismos para facilitar la reintegración al sistema educativo y al mercado laboral (Jacobo Suárez, 2017). Siete de cada diez mexicanos que regresaron de Estados Unidos entre 2009 y 2014 tenían entre 18 y 45 años de edad. La edad promedio de los hombres era de 37 años y para las mujeres era de 35.5 años de edad. En ambos casos, la mayoría estaban casados o vivían con su pareja (Fundación Bancomer BBVA, 2016).

En segundo lugar, el retorno reciente, aquel que ha ocurrido en el siglo XXI, está caracterizado por migrantes que han vivido largos periodos en Estados Unidos, lugar que una mayoría considera como su residencia (París, 2010). A diferencia de los migrantes circulares, que mantienen aspectos prioritarios de su vida en la comunidad de origen, los migrantes de retorno reciente optaron por establecer su vida familiar, laboral y educativa en territorio estadounidense. Lo anterior implica que los migrantes deportados y sus familiares que los siguen en el exilio, son dramáticamente desarraigados de sus vidas y experimentan traumas, discriminación y, como veremos más adelante, múltiples obstáculos para reintegrarse a la vida social y económica del país de origen (Hagan y Rodríguez, 2001).

De acuerdo con varios estudios (MATT, 2013; González-Barrera, 2015; Fundación Bancomer BBVA, 2016), la principal razón por la que los migrantes mexicanos regresan desde Estados Unidos es la reunificación familiar (51\% de las mujeres y $41 \%$ de los 
hombres). En contraste, el retorno forzado es declarado como principal causa de retorno por 15 por ciento de los hombres y 4 por ciento de las mujeres. Sin embargo, es probable que las encuestas subestimen a los migrantes que fueron deportados. Algunos estudios cualitativos han encontrado que se requiere más de un encuentro entre el investigador y el migrante para que éste mencione una deportación, de haber existido (Medina y Menjívar, 2015).

Debido al estigma asociado a la deportación, muchas personas prefieren ocultarla porque en sus comunidades es percibida como un "fracaso migratorio". En realidad, existe un vínculo entre deportación y reunificación familiar como causa de retorno, pues quienes regresan para reunirse con su familia muchas veces lo hacen siguiendo a un familiar que ha sido forzado a salir de Estados Unidos. Esto es consistente con las cifras mencionadas previamente, donde la mayor parte de los deportados son hombres, y la mayor parte de las personas que regresan por motivos de reunificación familiar son mujeres.

Para esta población, la búsqueda de empleo y la posibilidad de tener y/o continuar con su educación superior son actividades prioritarias. Algunos estudios muestran que la población retornada se inserta en el mercado laboral relativamente rápido (García y Gaspar, 2017; MATT, 2013). Alrededor de 70 por ciento encuentra trabajo en los primeros tres meses después de su regreso (Ordaz y Li, 2013; Fundación Bancomer BBVA, 2016). Empero, la reintegración laboral va más allá de obtener un empleo. De manera integral, tendría que brindar seguridad social y la posibilidad de movilidad social, lo cual parece no suceder con la población retornada. Alrededor de un tercio de los hombres retornados son autoempleados y 60 por ciento trabaja como subordinado, indicando un bajo perfil en la reintegración laboral. Además, una parte importante de la población de retorno entra al mercado laboral a través del sector informal, donde 30 por ciento encuentra su primer empleo al regreso y donde carecen de seguridad social (Fundación Bancomer BBVA, 2016).

Otro desafío laboral es el hacer uso de la experiencia adquirida en Estados Unidos, y continuar desarrollando habilidades profesionales en México (MATT, 2013; García Zamora, 2014; García y Gaspar, 2017). En general, los migrantes de retorno no se insertan en el sector en el que tienen mayor experiencia laboral. Mientras que en Estados Unidos la mayor parte de los migrantes trabajaba en la industria de la construcción, o en el sector de servicios (educativos, salud, hotelería y restaurantes), en México los hombres retornados se concentran en la agricultura y la pesca (62\%), mientras que las mujeres se emplean en el sector de ventas y en el de servicios (60\%) (Gandini et al., 2015). Así, los estudios sugieren que en el proceso de reintegración laboral, el desafío principal es insertarse al mercado en condiciones laborales no precarias.

La documentación es otra de las necesidades básicas para los retornados y sus familias, sin importar la edad y el lugar de nacimiento. Irónicamente, los retornados encuentran múltiples dificultades para demostrar legalmente que son mexicanos, lo que constituye una barrera para la obtención de servicios de toda índole y, por tanto, para su reintegración. Los 
migrantes que son deportados no tienen la oportunidad de planear su regreso y frecuentemente carecen de documentos básicos en México, por ejemplo, documentos de identidad y constancias de estudios realizados en Estados Unidos. Muchos migrantes de retorno no pueden viajar a Estados Unidos para conseguirlos, por lo menos en el corto plazo. De acuerdo con la organización Mexicans and Americans Thinking Together (MATT, 2013, 40 por ciento de los mexicanos que regresaron en el 2015 carecía de algún documento que probara su nacionalidad. A los adultos en retorno, se suman los menores de edad. Dentro de este último grupo, existen alrededor de medio millón de niños y niñas mexicano-americanos -matriculados en primaria- quienes tienen plenos derechos tanto en México como en Estados Unidos, por ser ciudadanos de ambos países.

Para los menores de 18 años, la incorporación y/o la continuidad de su educación básica, secundaria y bachillerato, según sea el caso, constituye una necesidad inmediata. El acceso a la educación está directamente relacionado con la documentación. Los niños y jóvenes retornados enfrentan diversos retos para acceder a las escuelas en México, desde revalidar sus estudios realizados en los Estados Unidos para continuar su educación en México, hasta utilizar el español, y ya no el inglés, como lengua para el aprendizaje en el aula, entre otros. Los problemas de documentación son más complicados para los hijos de inmigrantes mexicanos nacidos en Estados Unidos, ya que las autoridades mexicanas usualmente requieren apostillas y traducciones oficiales de sus certificados de nacimiento para que puedan acceder a los servicios de salud, matricularse en la escuela o revalidar sus estudios (Jacobo Suárez y Landa, 2015).

\section{LA RESPUESTA DEL GOBIERNO FEDERAL MEXICANO (2006-2017)}

Una vez dimensionado el flujo de retorno reciente a México, y habiendo detallado las necesidades de esta población, surge la incógnita de cómo ha sido el proceso de reintegración de los migrantes a México, ya sea a sus comunidades de origen o a un nuevo destino. En particular, nos interesa analizar los esfuerzos del gobierno federal en materia de reintegración de migrantes mexicanos y cómo han funcionado hasta ahora. Las administraciones de Felipe Calderón (2006-2012) y Enrique Peña Nieto (2012-2018) consideran el retorno migratorio en dos niveles. Por una parte, durante sus administraciones se incluyeron dentro de leyes y reglamentos específicos los derechos de los migrantes retornados y sus familias. Por otra parte, ambas administraciones establecieron programas y líneas de acción, algunas veces dentro de las leyes creadas, con el fin de regular no solo la recepción, sino también la reintegración de las personas repatriadas. No ha existido un orden específico para la creación de estos instrumentos, por lo que en ocasiones un programa puede preceder a la creación de leyes.

Durante la administración del presidente Felipe Calderón se dieron algunos pasos favorables para regular la migración, incluyendo la creación de la primera Ley de Migración 
a nivel nacional, y su reglamento. Incluso se aprobaron varias leyes a nivel federal y local para regular otros aspectos relacionados con el tema migratorio. La Ley de Migración de 2011 (DOF, 2011) es el primer esfuerzo para construir un marco regulatorio en materia migratoria en México. En esta sección analizaremos dicha Ley y algunos programas federales de apoyo para la población retornada. Discutimos primero la Ley de Migración y, posteriormente, los programas debido a que la Ley establece un marco general y los programas se enfocan a la implementación de acciones específicas.

Anterior a la creación de la Ley de Migración, los temas migratorios eran regulados por la Ley General de Población. No obstante, la nueva legislación es más bien una ley de Inmigración (de extranjería) (Calderón, 2012), pues excluye aspectos importantes de la emigración y el retorno. Con respecto al retorno, la Ley hace referencia mínima y menciona que se ha de "facilitar el retorno a territorio nacional y la reinserción social de los emigrantes mexicanos y sus familias a través de programas interinstitucionales y de reforzar los vínculos entre las comunidades de origen y destino de la emigración mexicana, en provecho del bienestar familiar y el desarrollo nacional y regional" (DOF, 2011, s/n).

El tema del retorno es discutido de manera más amplia en las modificaciones a la Ley de Población hechas en 2012, específicamente dentro del Reglamento de la Ley, donde se regula por primera vez las condiciones de repatriación y recepción de mexicanos, así como las instancias responsables de dichos procesos (Capítulo 10). En los artículos 216 y 218 se menciona que la Secretaría de Gobernación, por conducto del Instituto Nacional de Migración, tomará las medidas y acciones necesarias para la recepción y atención de los mexicanos repatriados.

Asimismo, se estipula que la Secretaría ofrecerá en los lugares destinados para la recepción de mexicanos repatriados diversos servicios en coordinación con otras dependencias del gobierno federal, estatal y municipal, así como con organismos, instituciones y empresas de los sectores público, privado y social. Los servicios incluyen información y orientación básica sobre los servicios de apoyo ofrecidos, alimentación para las necesidades inmediatas básicas; comunicación con el consulado mexicano, en caso de que un repatriado desee hacer una denuncia en contra de la autoridad migratoria extranjera derivada de su proceso de traslado a México; así como asistencia médica y psicológica, llamadas telefónicas; canalización hacia albergues temporales y traslados del punto de repatriación hacia la comunidad de origen del migrante. Asimismo, se establece que la autoridad migratoria ha de entregar una constancia a todo mexicano que pruebe su ingreso al país.

Dentro de las modificaciones de 2012 también se estableció que la Secretaría de Gobernación, en coordinación con la Secretaría de Relaciones Exteriores, podría suscribir acuerdos interinstitucionales con otros países y organismos internacionales en materia de 
repatriación segura, digna y ordenada, vigilando que se respeten los derechos de los mexicanos repatriados en los lugares de recepción. ${ }^{3}$

La Ley y su reglamento no abordan de manera extensa la reintegración de los migrantes, ni las medidas orientadas a consolidar dicho proceso. En su lugar, el artículo 218 bis del Reglamento establece que la Secretaría de Gobernación celebrará acuerdos de coordinación con las dependencias y entidades de la Administración Pública Federal, estatal y municipal, así como con organismos e instituciones públicas y privadas para la reintegración de los mexicanos repatriados. Dichos acuerdos pueden considerar programas de atención médica, acciones para orientar a las personas, proporcionándoles información, sobre ofertas de empleo y vivienda y creación de proyectos de coinversión; también debe promover la instauración de albergues para mexicanos repatriados, así como la creación de programas de difusión para informar a los mexicanos indígenas repatriados sobre sus derechos, procurando que esta información se emita en su lengua de origen (DOF, 2012).

Además de la creación de leyes y marcos regulatorios, la administración de Felipe Calderón creó el Fondo de Apoyo a Migrantes (FAM) en 2009. Este fondo fue creado de manera emergente como un subsidio bajo el ramo 23 del Presupuesto de Egresos de la Federación (PEF) con el fin de "apoyar a los trabajadores migrantes en retorno y a las familias que reciben remesas para que puedan encontrar una ocupación en el mercado formal, cuenten con opciones de autoempleo, generen ingresos y mejoren su capital humano y vivienda" (DOF, 2012, p. 1). Dado que los recursos del Fondo tienen el carácter de subsidio, deben ser destinados a proyectos, acciones y obras de infraestructura, mismos que les servirían a los trabajadores en retorno para encontrar empleo en el mercado formal o autoempleo; para generar ingresos, mejorar su capital humano y su vivienda, apoyar la operación de los albergues que los atienden y para ayudar a regresar a sus comunidades de origen (Osorno, 2014). Los recursos del fondo son destinados a 24 entidades federativas, específicamente a municipios caracterizados por su marginación, altos índices de migración y con una dependencia importante de las remesas. Para la operación de los recursos están involucradas diversas dependencias a nivel federal y estatal tales como la Secretaría de Hacienda y Crédito Público, la Secretaría de Finanzas, la Secretaría de la Contraloría y los gobiernos locales.

Al iniciar la administración de Enrique Peña Nieto, el principal cambio en materia migratoria fue bajar el tema a un nivel programático y en líneas de acción específicas, aunque

\footnotetext{
${ }^{3}$ Los derechos a los que se hace referencia en el artículo 84 de la Ley de Población son: acceder a comunicación telefónica; recibir agua y alimentos, un espacio digno, enseres básicos para su aseo personal y atención legal, psicológica y médica; ser informado respecto de los diversos programas y apoyos que puede recibir; no ser discriminado por las autoridades a causa de su origen étnico, sexo, género, edad, discapacidad, condición social, entre otros (DOF, 2018, pp. 8-9).
} 
con importantes limitaciones presupuestales y operativas. La migración de retorno se incluyó en el Plan Nacional de Desarrollo (2013-2018) a través del eje 5: "México con responsabilidad Global". Dicho eje establece objetivos, estrategias y líneas de acción para la atención de las necesidades de las personas migrantes y sus familias en todas las fases del proceso migratorio, incluyendo la migración de retorno. Por ejemplo: hacer una revisión y reforzar los acuerdos de repatriación de los mexicanos, así como la creación y fortalecimiento de programas de certificación de habilidades y reinserción laboral, social y cultural para las personas migrantes de retorno a sus comunidades de origen, entre otros. Posteriormente, en 2014 se dio un avance significativo en materia migratoria con la creación del programa Especial de Migración (PEM). El PEM va en concordancia con el Plan Nacional de Desarrollo y contempla estrategias y acciones para regular la migración de manera integral, intersectorial y atendiendo a diversas dimensiones del fenómeno migratorio. En realidad, el PEM fue presentado como el eje central de la política y acciones en materia migratoria. De acuerdo con la Secretaría de Gobernación:

El PEM es la base para la instrumentación de una política de Estado en materia migratoria, con un enfoque de atención integral y adecuada gobernanza de las distintas dimensiones de la migración en México como país de origen, tránsito, destino y retorno de migrantes.

Por su carácter transversal, el PEM contribuye al logro de las cinco metas nacionales establecidas en el Plan Nacional de Desarrollo 2013-2018 y establece las bases para la articulación de los esfuerzos de la Administración Pública Federal en la materia, la coordinación con otros poderes y órdenes de gobierno, y la participación de la sociedad civil (Secretaría de Gobernación, 2015, s/n).

Además de responder al fenómeno de manera integral, el PEM buscaba impulsar una política migratoria participativa. El propio programa fue elaborado después de un proceso de concertación entre el gobierno, la academia y organizaciones de la sociedad civil. Ochenta organizaciones participaron en el PEM y se hicieron llamar el Colectivo Plan Nacional de Desarrollo, lo que permitió incluir un diagnóstico migratorio, cinco objetivos y 195 líneas de acción (Ramos, 2015). Uno de estos objetivos hace referencia explícita por primera vez a la migración de retorno.

Así, el PEM busca "Favorecer los procesos de integración y reintegración de las personas migrantes y sus familiares" (objetivo 4). En congruencia con este objetivo, la estrategia 4.6 plantea desarrollar esquemas de atención especializada para la integración de personas migrantes, repatriadas, refugiadas y quienes reciben protección complementaria. De manera específica, se contempla la creación de mecanismos de integración para los repatriados en varios ámbitos tales como el económico, social, cultural, de reinserción laboral a nivel local articulados con los programas de repatriación. A pesar del avance que representó la creación del PEM en términos programáticos, el primer gran obstáculo para la consecución de sus objetivos ha sido la ausencia de presupuesto asignado a este. 
Previo a la elaboración de leyes y programas en materia migratoria, se firmaron los primeros acuerdos entre México y Estados Unidos, como el Memorándum de Entendimiento entre la Secretaría de Gobernación, la Secretaría de Relaciones Exteriores y el Departamento de Seguridad Interna de Estados Unidos (2004), y el Arreglo local para la repatriación de mexicanos entre California y Baja California (2008), para regular los puntos de recepción, horarios y garantías en el proceso de deportación. Con base en estos primeros acuerdos se creó el programa de Repatriación Humana (PRH) en el 2007 y, posteriormente, fue reemplazado por el programa Somos Mexicanos en 2013. Desde un principio el programa fue implementado por el Instituto Nacional de Migración (INM) con el objetivo de coordinar diversas iniciativas para asistir a la recepción y reintegración de la población deportada.

El programa Somos Mexicanos ofrece una amplia gama de servicios de recepción (Instituto Nacional de Migración, 2015). Rietig y Domínguez (2015) distinguen los servicios de recepción como acciones en el corto plazo para atender a los retornados una vez que llegaron a territorio mexicano (por ejemplo, ofrecerles comida, agua, llamadas telefónicas y transporte) de los programas de reintegración, aquellos que brindan servicios de largo plazo con el fin de ayudarles a los retornados a establecerse y adaptarse en sus comunidades. Así, los centros de repatriación se enfocan primordialmente a servicios de recepción y en menor medida, ofrecen servicios de asesoría y trámites legales orientados a la reintegración. En este último tipo de servicios intervienen otras subsecretarías de la Secretaría de Gobernación, así como Secretaría de Relaciones Exteriores (Instituto Nacional de Migración, 2015).

También se proveen servicios de asistencia médica e información sobre opciones para continuar con sus estudios, como por ejemplo trámites de revalidación y equivalencias en caso de que el migrante tenga estudios inconclusos realizados en Estados Unidos y que desee retomar en una institución de educación superior en México. Se debe resaltar que las iniciativas incluidas en este programa no son implementadas por el INM, sino por una diversidad de dependencias federales. No están claro los mecanismos de coordinación entre dependencias y el INM, como tampoco en quién recae la responsabilidad última por los resultados del programa debido a que no hay una sola agencia que ejecute los recursos monetarios e implemente las acciones (Balanḉa, 2016).

\section{ANÁLISIS: ALCANCES Y LIMITACIONES DE LA RESPUESTA FEDERAL}

De acuerdo con la información presentada en las secciones previas, el gobierno mexicano ha aprobado diversas leyes y puesto en marcha programas y acciones para la protección de los migrantes retornados. Pareciera entonces que el gobierno federal está preparado para recibir a los mexicanos repatriados desde Estados Unidos. Sin embargo, la respuesta para atender los flujos de retorno forzado y voluntario ocurridos durante el presidente Barack Obama (2009-2016) y en los primeros meses de gestión de Donald Trump, ha sido tardía y de poca prioridad presupuestaria en la agenda nacional. Además, los servicios ofrecidos 
dentro los programas existentes parecen tener poca utilidad para atender las necesidades a las que se enfrenta la población retornada. Por lo tanto, hay varios retos en el proceso de garantizar una reintegración exitosa de la población retornada.

Durante la administración de Peña Nieto hubo un avance, al transitar de las leyes y reglamentos en materia migratoria -creados bajo la presidencia de Felipe Calderón- hacia programas y líneas de acción específicas para fomentar la reintegración social, económica y educativa de la población retornada.

Sin embargo, la creación de programas no es suficiente. Se requieren criterios y líneas de acción congruentes con el problema a resolver, un presupuesto adecuado, y la correcta implementación del programa en todos los niveles, además de definir responsabilidades y resultados asequibles y medibles. En todos estos casos existen desafíos a resolver. El programa Especial de Migración no ha tenido presupuesto asignado desde su creación, es decir, no se han asignado ni un peso para su operación. Somos Mexicanos y el Fondo de Apoyo al Migrante han recibido financiamiento, pero ha sido considerado insuficiente (Castaño, 2016; Fundar, 2016). Si bien un mayor presupuesto no garantiza la consecución de los objetivos, cuando los recursos son escasos se restringe la puesta en marcha de los programas y se reduce la población beneficiada.

Somos Mexicanos, fue el principal programa durante la administración de Peña Nieto para atender a la población retornada en términos laborales, de salud, documentación, educativos, entre otros, no cuenta con recursos etiquetados para su operación. ${ }^{4}$ En palabras del titular del INM, Somos Mexicanos es más una estrategia de operación, pues el presupuesto proviene de las unidades responsables de ejecutar los distinto componentes del programa. Por ejemplo, la Secretaría del Trabajo destina recursos para ejecutar Repatriados Trabajando, uno de los subprogramas de Somos Mexicanos. Y así, las demás agencias federales involucradas en el programa asignan recursos y ejecutan líneas de acción. El INM ha declarado que el presupuesto asignado por ellos para atender repatriados ha sido muy bajo.

En un informe que presentó el Instituto ante el Poder Legislativo para solicitar mayores recursos, se dio a conocer que el gasto en el 2012 para el programa fue de 11994681.12 pesos mexicanos, es decir, 43.88 pesos por persona retornada; cifra -en propias palabras del INM- muy reducida para cumplir con los objetivos planteados. El Instituto señaló que los recursos que necesitaría para cumplir cabalmente con el programa tendrían que ser de 584 625500 pesos mexicanos, cifra pensada para 400000 eventos de deportación anuales, es decir 1450 pesos por persona retornada (Instituto Nacional de Migración, 2013).

\footnotetext{
${ }^{4}$ En 2016 se solicitó tanto al INM como a la Cámara de Diputados el presupuesto anual asignado a Somos Mexicanos, con los gastos desagregados por rubros. En ambos casos, la respuesta fue que no hay recursos etiquetados para este programa por lo que su asignación puede variar de acuerdo con la disponibilidad de recursos.
} 
En el mismo sentido, los recursos asignados al FAM, han sufrido constantes modificaciones desde el 2009 y en los últimos años se han reducido (Tabla 1).

Tabla 1. Presupuesto del Fondo de Apoyo al Migrante 2009-2017

(en millones de pesos)

\begin{tabular}{llllllllll}
\hline Año & 2009 & 2010 & 2011 & 2012 & 2013 & 2014 & 2015 & 2016 & 2017 \\
Monto & 300 & 100 & 100 & 300 & 200 & 200 & 300 & 300 & 267 \\
\hline
\end{tabular}

Fuente: Elaboración propia con datos del PEF.

Además de presupuesto insuficiente, los programas actuales muestran limitaciones importantes en su diseño, específicamente en cuanto a cómo se concibe y define a la población de retorno. Los servicios ofrecidos bajo Somos Mexicanos están disponibles solo para aquellas personas que ingresaron al país por los puntos de repatriación y que presenten su constancia de repatriación, la cual tiene una validez de seis meses. Con este comprobante, excluyen a los migrantes que fueron deportados, pero que no fueron recibidos por el INM en los centros de repatriación, así como a aquellos que tienen más de seis meses en el país y que aún requieren de asistencia para conseguir un trabajo, documentación, etcétera. También se excluye a las personas cuyo retorno es considerado "voluntario", aun cuando ellos experimenten también dificultades severas para reintegrarse. En este grupo se incluye aquellos que siguen a un familiar deportado en su regreso a México, en general dependientes económicos de quien es deportado; y a aquellos que regresan de manera no planeada, debido a complicaciones de salud, y a migrantes que firman una orden voluntaria de retorno.

Se pensaría que al restringir la población objetivo se beneficiaría a un mayor porcentaje de esta. Sin embargo, de acuerdo con la Unidad de Política Migratoria, el alcance de Somos Mexicanos ha sido modesto. El porcentaje del total de las personas repatriadas que recibieron el apoyo del programa aumentó de 57 por ciento en el 2010 a 82 por ciento en 2015 . Sin embargo, el universo a atender disminuyó en casi 36 por ciento durante ese periodo pues en 2010 las personas repatriadas fueron un total de 496 268, mientras que para el 2015 la cifra bajó a 207398 (Tabla 2).

Tabla 2. Eventos de repatriación de mexicanos y apoyos otorgados por PRH/Somos Mexicanos (2010-2015)

\begin{tabular}{cccc}
\hline Año & $\begin{array}{c}\text { Eventos de } \\
\text { repatriación }\end{array}$ & $\begin{array}{c}\text { Beneficiarios del } \\
\text { programa }\end{array}$ & $\begin{array}{c}\text { Porcentaje de } \\
\text { cobertura }\end{array}$ \\
\hline 2010 & 469268 & 267317 & 56.9 \\
2011 & 405457 & 269197 & 66.3 \\
2012 & 369492 & 273300 & 73.9 \\
2013 & 332865 & 239932 & 72.1 \\
\hline
\end{tabular}




\begin{tabular}{llll}
\hline 2014 & 243196 & 198876 & 81.7 \\
2015 & 207398 & 171858 & 82.8 \\
\hline
\end{tabular}

Fuente: Elaboración propia con datos del Boletín estadístico de la Unidad de Política Migratoria (Secretaría de Gobernación/Unidad de Política Migratoria, 2010-2015).

Cabe señalar que Somos Mexicanos incluye una amplia gama de servicios y la información proporcionada no identifica el tipo de ayuda recibido. Ante la falta de apoyo proveniente del gobierno, los migrantes buscan ayuda en sus comunidades. El estudio realizado por MATT (2013) en Jalisco, muestra que 95.7 por ciento de los retornados no recibieron asistencia por parte del gobierno. Por el contrario, sus familiares y amigos fueron las principales fuentes de apoyo para obtener empleo, conseguir documentos, acceder a los sistemas de salud y educación, etcétera.

Unos de los componentes principales de Somos Mexicanos es el programa Repatriados Trabajando, implementado por la Secretaría del Trabajo y Previsión Social, y en particular, por el Servicio Nacional de Empleo. Su objetivo es apoyar a connacionales repatriados de los Estados Unidos para que regresen a su lugar de origen y obtengan empleo. El programa tiene dos fases. En la primera etapa, se apoya económicamente -hasta con 2500 pesos mexicanos- a los repatriados que hayan llegado a los estados fronterizos o al aeropuerto de la Ciudad de México para comprar un boleto de avión o de autobús que les permita regresar a su lugar de origen. Una vez que ya están en su lugar de origen o residencia, la segunda etapa consiste en darles orientación y brindarles información necesaria para la búsqueda de empleo. En caso de no existir vacantes, son canalizados a otros de los subprogramas del Programa de Acceso al Empleo (PAE). Asimismo, se les da un apoyo económico equivalente a un salario mínimo mensual, el cual se entrega en dos partes de acuerdo con requisitos específicos. El primer monto (medio salario mínimo mensual) se da siempre y cuando el solicitante acuda a las Oficinas del Servicio Nacional de Empleo en su lugar de origen o residencia, dentro de los veinte días hábiles posteriores a la fecha de su repatriación, y que haya ingresado por alguno de los puntos oficiales. La segunda parte del monto (medio salario mínimo mensual) se da cuando el migrante entrega evidencia de haberse presentado a todas las entrevistas de las vacantes o informe el estatus de su incorporación a alguno de los subprogramas del PAE.

Una vez en el lugar de origen, se otorga un apoyo complementario para la búsqueda de empleo de 500 a 1000 pesos, según sea el estado de destino. Se provee además información y orientación para la vinculación laboral.

Es importante señalar que los interesados en participar en el subprograma Repatriados Trabajando, deben estar registrados en los listados de eventos de repatriación del Instituto Nacional de Migración (INAMI) y/o en los Consulados de México en la frontera norte, con fecha máxima de 15 días previos a la fecha de presentarse al SNE para ser apoyados. En consecuencia, para ser elegible para esta ayuda, la persona repatriada ha de aplicar en un 
periodo muy corto a partir de su regreso a México. En términos de cobertura y cumplimiento de objetivos, Repatriados Trabajando tampoco ha tenido un amplio número de beneficiarios (Tabla 3).

Tabla 3. Apoyos y eventos de colocación. Repatriados trabajando (2005-2017)

\begin{tabular}{ccc}
\hline Año & Apoyos & Eventos de colocación \\
\hline 2005 & 144 & 26 \\
2006 & 1463 & 694 \\
2007 & 6701 & 1355 \\
2008 & 21171 & 4890 \\
2009 & 19093 & 7377 \\
2010 & 27621 & 776 \\
2011 & 26649 & 396 \\
2012 & 26488 & 293 \\
2013 & 10143 & S/D \\
2014 & 12669 & S/D \\
2015 & 3820 & S/D \\
2016 & 6769 & S/D \\
2017 & 60 & S/D \\
\hline
\end{tabular}

Fuente: Secretaría del Trabajo y Previsión Social/Subprograma de Apoyo al Empleo (2005-2017).

El bajo número de personas retornadas beneficiadas por los programas ofrecidos por el gobierno federal se debe a varias razones. El desconocimiento de la existencia de los programas por parte de los retornados es común, lo que nos lleva a cuestionar la difusión y estrategia de comunicación por parte del gobierno. Un estudio llevado a cabo en el punto de repatriación ubicado en Tijuana muestra que 92 por ciento de las personas encuestadas no tenían conocimiento alguno del programa Somos Mexicanos y los servicios que este ofrece, aun cuando se les entrevistó justo después de ser recibidos por el personal del Instituto Nacional de Migración. De acuerdo con esta investigación, lo anterior se podría explicar porque el estado psicológico en que retornan los connacionales les impide retener la información que las autoridades migratorias mexicanas les proveen al recibirlos en el módulo de repatriación. De ser cierto esto, una alternativa es proporcionar la información de manera reiterada desde que el migrante regresa al país. Otra hipótesis es que la información acerca del programa y sus beneficios no es transmitida de manera clara y concisa (López, 2012). Estas situaciones no son excluyentes, pueden coexistir y reforzar la poca y pobre información que reciben los migrantes sobre programas de ayuda en su retorno.

Otro aspecto a señalar es que los proyectos apoyados y desarrollados con los recursos del FAM han sido para obras de infraestructura, como pavimentación, instalación de sistemas 
hidráulicos. Lo anterior está establecido en las propias reglas de operación del programa. Sin embargo, es importante cuestionar si llevar a cabo programas de infraestructura le servirá a la población de retorno para encontrar empleo, generar ingresos y cumplir con los objetivos del propio Fondo, mencionados previamente.

Además de las dificultades ya mencionadas, otro desafío para atender a la población retornada es implementar acciones que respondan a sus necesidades más apremiantes. Desde hace años, una petición constante de los retornados ha sido obtener un documento emitido por el gobierno de México que avale su identidad personal, pues resulta un requisito indispensable para acceder a cualquier servicio en México. Sin una identificación oficial, el trámite más básico, como contratar servicios de telefonía, rentar una vivienda, o abrir una cuenta bancaria es imposible. Obviamente, ingresar al sistema educativo o de salud es prácticamente inviable si el individuo no puede identificarse legalmente como mexicano. Quienes retornan de manera forzada, generalmente carecen de documentos de identidad, como la credencial de elector expedida por el Instituto Nacional Electoral (INE) o el acta de nacimiento mexicana.

El gobierno federal mexicano ha respondido de manera parcial y tardía a este problema al firmar un convenio con los gobiernos de algunos estados de Estados Unidos para poder otorgar el acta de nacimiento mexicana a hijos de migrantes mexicanos nacidos en ese país. Este convenio se firmó en septiembre de 2016, a pesar de que desde el 2010 algunos estudios habían identificado alrededor de 600000 niños mexicano-americanos residiendo en México, muchos de ellos con problemas de documentación (Escobar, et al. 2013). No existe información pública respecto al número de registros de nacionalidad mexicana realizadas desde septiembre de 2016.

Los migrantes que son repatriados pueden recibir la constancia de repatriación", que los hace acreedores de los servicios del programa Somos Mexicanos. Sin embargo, no es una identificación válida para resolver algunas de las primeras necesidades que tienen los retornados al llegar a territorio mexicano, tales como cambiar dólares por pesos mexicanos o abrir una cuenta de banco (Rendón y Wertman, 2017). La credencial de elector, expedida por el Instituto Nacional Electoral (INE), es probablemente la identificación oficial más aceptada y requerida para los mayores de edad. Resulta inverosímil que aún en el 2017 años después de la mayor entrada de retronados al país- aún no existían módulos del INE en los puntos de recepción de los repatriados.

\section{CONCLUSIONES: RECOMENDACIONES DE POLÍTICA PÚBLICA}

Con base en análisis presentado, concluimos que los programas y apoyos ofrecidos por el gobierno federal a la población migrante de retorno requieren basarse en una definición de 
población retornada más amplia. Limitar los programas de asistencia al retorno a quienes han pasado por un proceso legal de repatriación, es inadecuado y restrictivo. El apoyo ha de estar disponible a cualquier migrante que haya regresado de Estados Unidos, independientemente de si fue deportado o regresó voluntariamente.

En segundo lugar, recomendamos ampliar sustantivamente el periodo en el que se otorgan los apoyos. Bajo el programa Somos Mexicanos, los apoyos se entregaban solo durante los primeros seis meses posteriores al regreso a México. Sin embargo, el retorno no es un punto en el tiempo, sino un proceso individual que puede durar meses o años, dependiendo de la experiencia y las características de cada migrante, por lo que se requiere desarrollar programas de largo plazo que otorguen apoyo por plazos más extensos.

En particular, sugerimos priorizar programas de reintegración que aseguren la obtención de documentos de identidad oficiales. La credencial de elector debe ser ofrecida y tramitada de forma expedita. Igualmente importante es proveer atención psicológica, en particular a aquellos migrantes que residieron por largos periodos en Estados Unidos, incluyendo a aquellos que experimentaron una deportación, encarcelamiento, o separación familiar. Los servicios de salud mental han estado ausentes dentro de la estrategia del gobierno federal, ignorando así la relevancia que emociones como traumas, tristeza, enojo, impotencia y dolor imprimen al proceso de reintegración.

En tercer lugar, recomendamos asignar presupuesto suficiente a los programas de atención a la población retornada, así como definir los objetivos que han de alcanzar estos. La política de atención por parte del gobierno federal hacia la población que retorna tiene que ir acompañada de presupuesto suficiente y constante, como mínimo, para poder operar. Cabe resaltar que durante la actual administración del presidente Andrés Manuel López Obrador (2018-2024) se redujeron significativamente los programas y apoyos a la población migrante en general, y en particular a los programas de apoyo al retorno, debido al plan de austeridad del gasto público. En este sentido, es importante recuperar un piso mínimo de apoyo social y económico para esta población por parte del gobierno federal. Finalmente, enfatizamos la importancia de realizar evaluaciones públicas de los programas ya implementados para identificar aquellos elementos de éxito que podrían ser replicados, así como identificar estrategias que son susceptibles de mejora. Es importante aprender de las acciones realizadas por las administraciones previas para construir mejores programas y estrategias en el presente.

\section{REFERENCIAS}


\begin{tabular}{l|l}
20 & $\begin{array}{l}\text { Back on your own: migración de retorno y la respuesta del gobierno federal en México } \\
\text { Jacobo Suárez, M.L. y Cárdenas Alaminos, N. }\end{array}$
\end{tabular}

Alanís Enciso, F. S. (2015). Voces de la Repatriación. En La sociedad Mexicana y la repatriación de mexicanos de Estados Unidos 1930-1933. México: El Colegio de San Luis/El Colegio de la Frontera Norte/El Colegio de Michoacán.

Balança, I.L. (2016). Los desafios de la coordinación intergubernamental: la atención a migrantes mexicanos deportados en el estado de Baja California. (Tesis de maestría). El Colegio de la Frontera Norte, Tijuana.

Calderón Chelius, L. (2012). Cambios en la agenda migratoria: entre el nuevo marco normativo y las nuevas formas de migración en México. En T. Ramírez García y M.A. Castillo (Coords.), México ante los recientes desafíos de la migración internacional. (pp.19-50 ). México: Consejo Nacional de Población.

Castaño, P. (19 de abril de 2016). Desfavorable el presupuesto 2016 para la atención a personas migrantes y refugiadas [publicación en línea]. Recuperado de http://fundar.org.mx/desfavorable-el-presupuesto-2016-para-la-atencion-a-personasmigrantes-y-refugiadas/

Department of Homeland Security. (2015). 2014 Yearbook of Immigration Statistics. Washington, D.C.: Office of Immigration Statistics. Recuperado de https://www.dhs.gov/sites/default/files/publications/DHS\%202014\%20Yearbook.pdf

Diario Oficial de la Federación. (2011). Ley de Migración. Ciudad de México, México, publicada el 25 de mayo de 2011.

Diario Oficial de la Federación. (2012). Reglamento de la Ley de Migración. Ciudad de México, 28 de septiembre de 2012.

Diario Oficial de la Federación, Ciudad de México, México. (2018) Ley General de Población. Publicada el 12 de julio de 2018. Recuperado de http://www.diputados.gob.mx/LeyesBiblio/pdf/140_120718.pdf

Durand, J. y Arias, P. (2014). Escenarios locales del colapso migratorio. Indicios desde los altos de Jalisco. Papeles de Población, 20(81), 165-192.

Durand, J. (2016). Historia Mínima de la migración México-Estados Unidos. México: El Colegio de México.

Durand, J. (2000). Tres premisas para entender y explicar la migración México-Estados Unidos. Relaciones. Estudios de historia y sociedad, XXI(83), 1-11.

Escobar Latapí, A., Lowell, L. and Martin, S. (2013). Diálogo Binacional sobre Migrantes Mexicanos en Estados Unidos y México. Reporte final. México: Georgetown, Centro de Investigaciones y Estudios Superiores en Antropología Social /Georgetown University.

Fundación Bancomer BBVA. (2016). Anuario de Migración y Remesas. México 2016. C. Serrano y M. Jaramillo (Coords). México: Fundación Bancomer/Secretaría de Gobernación-Consejo Nacional de Población. 
Fundar/Centro de Análisis e Investigación. (11 de marzo de 2016). Inconsistencias en el desempeño y gasto del Instituto Nacional de Migración [publicación en línea]. Recuperado de http://fundar.org.mx/inconsistencias-en-el-desempeno-y-gasto-delinstituto-nacional-de-migracion/

Gandini, L., Lozano Ascencio, F. y Gaspar Olvera, S. (2015). El retorno en el nuevo escenario de la migración entre México y Estados Unidos. México: Consejo Nacional de Población.

García Zamora, R. (2014). Los impactos y desafios del retorno de los migrantes mexicanos de Estados Unidos: Hacia un programa integral de apoyo integral de los migrantes y sus familias. Ponencia presentada el 14 de noviembre en el Seminario Permanente sobre Migración Internacional. Recuperado de http:/www.colef.mx/wpcontent/uploads/2014/11/PPT-Rodolfo-Garcia-Zamora.pdf

García, R. y Gaspar, S. (2017). Migración de retorno de Estados Unidos a seis estados de México. Hacia la reintegración familiar y comunitaria. R. García Zamora (Coord.), El retorno de los migrantes mexicanos de Estados Unidos a Michoacán, Oaxaca, Zacatecas, Puebla, Guerrero y Chiapas 2000-2012, (pp. 15-58) México: Miguel Ángel Porrúa/Universidad Autónoma de Zacatecas.

González-Barrera, A. (19 de noviembre de 2015). More Mexicans leaving than coming to the U.S. Pew Research Center [publicación en línea]. Recuperado de http://www.pewhispanic.org/2015/11/19/more-mexicans-leaving-than-coming-to-the-us/

Guzmán Elizalde, L. (2014). Estudio regional sobre políticas públicas de integración de migrantes en Centroamérica y México. México: Sin Fronteras.

Hagan, J. y Rodríguez, N. (2001). Social effects of mass deportation by the United States Government, 2000-2010. Ethnic and Racial Studies, 34(8), 1374-1391.

Hagan, J., Eschbach, K. y Rodríguez, N. (2008). U.S. Deportation Policy, Family Separation, and Circular Migration. International Migration Review, 42(1), 64-88.

Hazán, M. (2014). Understanding return migration to Mexico: Towards a comprehensive policy for the reintegration of returning migrants. Center for comparative immigration studies, (193), 3-54. Recuperado de en https://ccis.ucsd.edu/ files/wp193.pdf

Instituto Nacional de Migración. (2013). Proyecto presupuesto 2014. Recuperado de https://www.gob.mx/cms/uploads/attachment/file/12408/PRESENTACION_PPTO_201 4 _ Y_FORTALECIMIENTO_INSTITUCIONAL_2.pdf

Instituto Nacional de Migración. (2015). Guía de Procedimiento de Repatriación al Interior de México PRIM. México: Secretaría de Gobernación-Instituto Nacional de MigraciónINM. 
\begin{tabular}{l|l}
22 & Back on your own: migración de retorno y la respuesta del gobierno federal en México \\
Jacobo Suárez, M.L. y Cárdenas Alaminos, N.
\end{tabular}

Jacobo Suárez, M. (2017). De regreso a "casa” y sin apostilla: estudiantes mexicoamericanos en México. Sinéctica. (48), 1-18.

Jacobo Suárez, M. y Landa, N. (1 de Agosto de 2015). La exclusión de los niños que retornan a México. Nexos. Recuperado de http://www.nexos.com.mx/?p=25878

Jacobo Suárez, M. (2014). De ida y de vuelta. El impacto de la política migratoria estadounidense en México y su población retornada. Carta Económica Regional, 26(114), 66-91.

López, A. (2012). La deportación de mexicanos desde Estados Unidos: acción gubernamental y social en Tijuana, Baja California. (Tesis de Maestría). El Colegio de la Frontera Norte, Tijuana.

Rendón, E. y Wertman, L. (2017). Reintegración Migrante: un modelo social, económico y empático para el retorno. México: Senado de la República. Recuperado de https://www.senado.gob.mx/comisiones/derechos_humanos/docs/Reintegracion_Migran te.pdf

Masferrer, C. y Roberts, B. (2016). The Changing Patterns of Return Migration from the US to Mexico and their Policy Implications. En D. L. Leal y N. P. Rodríguez (Eds.), Migration in an Era of Restriction and Recession. Immigrants and Minorities, Politics and Policy, (pp. 235-258). Suiza: Springer International Publishing.

Mexicans and Americans Thinking Together (2013). The US/Mexican Cycle: End of an Era. México: MATT.

Medina, D. y Menjívar, C. (2015). The context of return migration: challenges of mixedstatus families in Mexico's schools. Ethnic and Racial Studies, 38(12), 2123-2139.

Ordaz, L. y Li, J. (6 de mayo de 2013). Perfil socioeconómico y de inserción laboral delos migrantes mexicanos de retorno. Presentación realizada en el Seminario Internacional sobre Migración de retorno. CISAN-UNAM, 6 y 7 de mayo, ciudad de México.

Osorno, R. (2014). Cuarto Reporte en Migración. Agenda Puebla Migrante. Presupuestos Públicos en Materia Migratoria. México: Observatorio Ciudadano sobre Políticas Públicas para Migrantes-Puebla/Iniciativa Ciudadana para la Promoción de la Cultura del Diálogo A.C.

París Pombo, M. (2010). Procesos de repatriación. Experiencias de las personas devueltas por las autoridades estadounidenses. Washington D.C.: Woodrow Wilson International Center for Scholars.

Passel, J., D’Vera, C. y González-Barrera, A. (23 de abril de 2012). Net Migration from Mexico falls to zero. Washington D.C.: Pew Research Center. [Publicación en línea] Recuperado de https://www.pewresearch.org/hispanic/2012/04/23/net-migration-frommexico-falls-to-zero-and-perhaps-less/ 
Ramos García, J. M. (2015). La política migratoria mexicana y la seguridad fronteriza con Estados Unidos: cambio y continuidad, 2012-2014. Región y Sociedad, XXVII(64). 351382.

Rietig, V. y Domínguez, R. (2015). Stopping the revolving door. Reception and Reintegration Services for Central American Deportees. Washington: Migration Policy Institute.

Secretaría de Gobernación. (28 de mayo de 2015). Programa Especial de Migración (20142018). [Mensaje en página electrónica ]. Recuperado de https://www.gob.mx/segob/acciones-y-programas/programa-especial-de-migracionpem-2014-2018-5887

Secretaría de Gobernación/Unidad de Política migratoria. (2010-2015). Boletines estadísticos. Repatriación de Mexicanos. Cuadros 5.6 y 5.6.1 del 2010 al 2015. [Publicación en línea] Recuperado de http://www.politicamigratoria.gob.mx/es/PoliticaMigratoria/CuadrosBOLETIN?Anual= $2015 \& \operatorname{Secc}=5$

Secretaría de Gobernación/Unidad de Política Migratoria. (2004-2016). Boletines estadísticos anuales. Repatriación de Mexicanos. Cuadros 5.1 del 2004 al 2016. [Publicación en línea] Recuperado de http://www.politicamigratoria.gob.mx/es/PoliticaMigratoria/CuadrosBOLETIN?Anual= $\underline{2016 \& \mathrm{Secc}=5}$

Secretaría del Trabajo y Previsión Social y Subprograma de Apovo al Empleo. (2005-2017). Datos del Programa de Apoyo al Empleo/Repatriados Trabajando. [Publicación en línea] Recuperado de https://datos.gob.mx/busca/dataset/programa-de-apoyo-al-empleorepatriados-trabajando.

The New York Times (5 de septiembre de 2014). The Secure Communities illusion. The New York Times. Recuperado de http://www.nytimes.com/2014/09/06/opinion/thesecure-communities-illusion.html?_r=0

Transactional Records Access Clearinghouse (TRAC). (2013). Secure Communities and ICE deportations: a failed program? TRAC Series on ice deportation. [Publicación en línea] Recuperado de https://trac.syr.edu/immigration/reports/349/

Fondo de las Naciones Unidas para la Infancia/Save the Children/Red Latinoamericana de Acogimiento Familiar (2015). Niños, niñas y adolescentes retornados. Un análisis de los contextos y las respuestas de los servicios y las politicas de protección en El Salvador, Guatemala, Honduras y México. Argentina: El Fondo de las Naciones Unidas para la Infancia/Save the Children/Red Latinoamericana de Acogimiento Familiar.

World Economic Forum. (3 de marzo de 2019). Which countries have the most immigrants? Agenda. [Publicación en línea] Recuperado de 
24 Back on your own: migración de retorno y la respuesta del gobierno federal en México

Jacobo Suárez, M.L. y Cárdenas Alaminos, N.

https://www.weforum.org/agenda/2019/03/which-countries-have-the-most-immigrants$\underline{51048 \mathrm{ff1f9/}}$ 